\title{
Intraductal malignant tumors in the liver mimicking cholangiocarcinoma: Imaging features for differential diagnosis
}

\author{
Ah Yeong Kim and Woo Kyoung Jeong \\ Department of Radiology and Center for Imaging Science, Samsung Medical Center, Sungkyunkwan University School of Medicine, \\ Seoul, Korea
}

Keywords: Bile duct tumor; Intrabiliary metastasis; Intraductal growing hepatocellular carcinoma; Gadoxetic acidenhanced MRI

\section{INTRODUCTION}

The spectrum of bile duct lesions ranges from benign tumors, such as adenomas, papillomas, villous tumors and leiomyomas, to malignant lesions, such as invasive intraductal papillary neoplasm of the bile duct (IPNB) and intraductal cholangiocarcinomas. Bile duct tumors cause bile duct obstruction with biliary stasis and consequent symptoms such as jaundice, itching, weight loss and abdominal pain. Although diagnostic accuracy of imaging technique for bile duct tumors has been improved including computed tomography (CT), magnetic resonance imaging (MRI) and MR cholangiopancreaticography (MRCP), accurate differential diagnosis is sometimes difficult in real clinical practice. Hepatocellular carcinoma with bile duct invasion or intraductal growth of liver metastasis is a very rare disease, but accurate imaging diagnosis is important because misdiagnosis can result in wrong treatment strategy and can cause serious problem of patient-doctor relationship. Thus, to avoid such a tragedy, doctors should guess the variable differential diagnoses of bile duct malignancies which seems to be like intrahepatic cholangiocarcinoma. Herein, we report two rare cases of bile duct tumors.

\section{CASE REPORT}

\section{Case 1}

A 43-year-old male patient underwent total colectomy for multiple carcinomas in ascending and transverse colon, followed by adjuvant chemotherapy. According to the pathologic report, the pathologic T stage was $\mathrm{T} 2$ and $\mathrm{T} 1$, for ascending and transverse colon cancer, respectively and N stage was N1c, and tumors showed mucin production (10\%). There was no evidence of other organ metastasis on the initial imaging work up. However, two years later, segmental intrahepatic bile duct dilatation in liver segment 2 (S2) was depicted on the postoperative follow up abdominal computed tomography (CT) scan and there was $4 \mathrm{~cm}$ low density mass lesion around the dilated bile duct at the $\mathbf{S} 2$ periphery

\section{Abbreviations:}

ADC, apparent diffusion coefficient; AFP , a-fetoprotein; ALT, alanine aminotransferase; AST, aspartate aminotransferase; CA, carbohydrate antigen; CEA, carcino-embryonic antigen; CK, cytokeratin; CT, computed tomography; DWI, diffusion-weighted images; HCC, hepatocellular carcinoma; IPNB, intraductal papillary neoplasm of the bile duct; $\mathrm{MRCP}$, magnetic resonance cholangiopancreaticography; MRI, magnetic resonance imaging; SI, signal intensity; T1WI, T1-weighted images; T2WI, T2-weighted images

\section{Corresponding author : Woo Kyoung Jeong}

Department of Radiology and Center for Imaging Science, Samsung Medical Center, Sungkyunkwan University School of Medicine, 81 Irwonro, Gangnam-gu, Seoul 06351, Korea

Tel: +82-2-3410-1923, Fax: +82-2-3410-0049

E-mail: jeongwk@gmail.com 

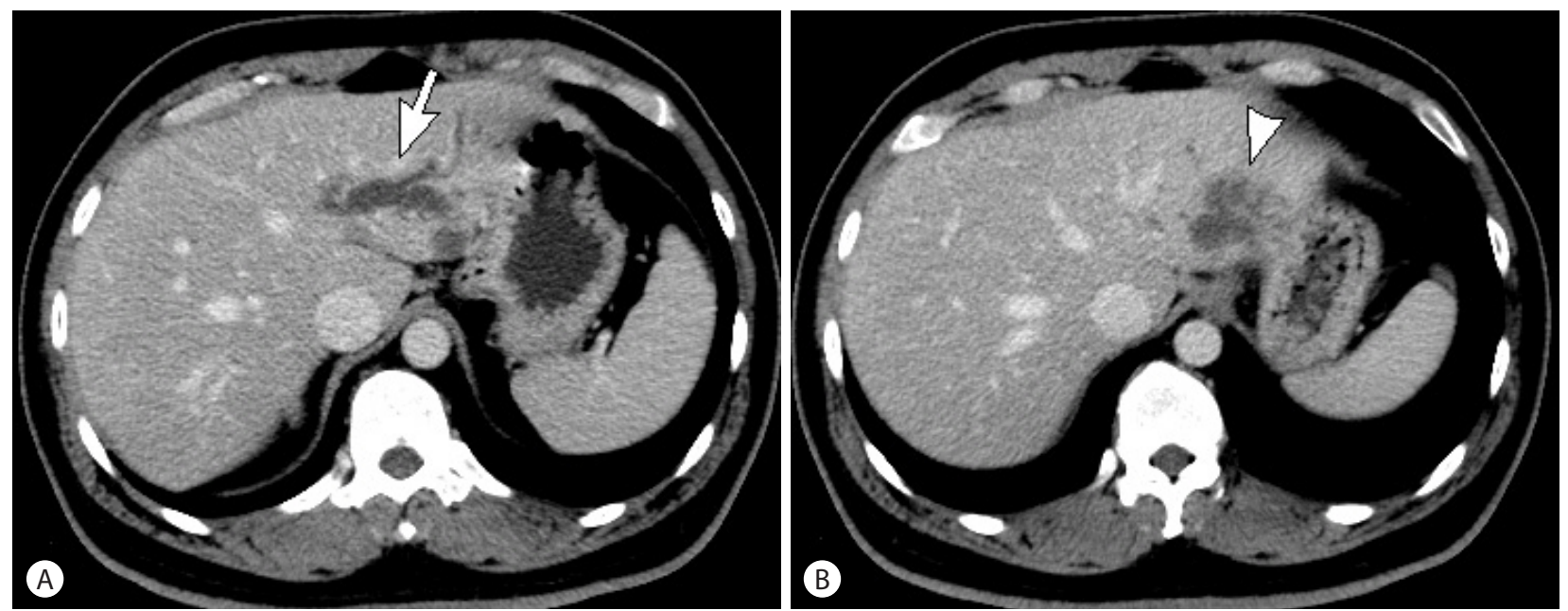

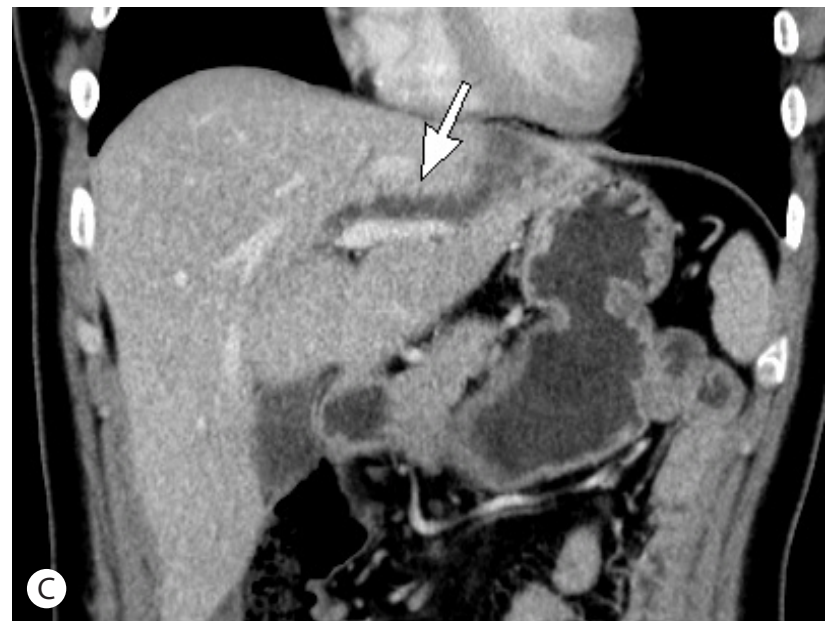

(Fig. 1). For further workup, Gadoxetic acid-enhanced pancreas magnetic resonance imaging (MRI) was performed. We again noted dilated bile duct in $\mathbf{S} 2$ with peripheral mass formation (Fig. 2). Within the dilated bile duct, intraductal lesion which showed high signal intensity (SI) on T2-weighted images (T2WI) and low SI on T1-weighted images (T1WI) was identified. After contrast administration, both the intraductal lesion and the peripheral mass did not show avid arterial enhancement and persistent low SI on the delayed phase. The mass demonstrated high SI on diffusionweighted images (DWI) and low apparent diffusion coefficient $(A D C)$ value. The serum tumor marker, carbohydrate antigen (CA) 19-9 was elevated up to $310.61 \mathrm{U} / \mathrm{mL}$, and carcino-embryonic antigen (CEA) was $15.06 \mathrm{ng} / \mathrm{mL}$. Our first impression was invasive intraductal papillary neoplasm of the bile duct (IPNB), however intraductal metastasis from colon cancer was also considered. He underwent left lateral segmentectomy. However, the histologic diagnosis was metastatic adenocarcinoma from colon with exuberant mucin production and extensive necrosis (40\%).
Figure 1. Segmental intrahepatic bile duct dilatation (white arrows on A, C) with low density mass lesion around the dilated bile duct at liver $\$ 2$ (white arrowhead on B) on the abdominal CT scan. CT, computed tomography.

\section{Case 2}

A 55-year-old male patient was referred to our hospital for evaluation of a hepatic tumor. The patient had alcoholic liver disease, and symptoms including jaundice and itching sense had been developed one week ago. Liver function tests showed normal range of aspartate aminotransferase (AST) and alanine aminotransferase (ALT). The serum tumor marker, $\alpha$-fetoprotein (AFP) was also normal range. However, CA 19-9 was elevated up to $465.85 \mathrm{U} / \mathrm{mL}$. There was an intraluminal growing polypoid enhancing mass at the primary confluence of bile duct, extending to the second order branch of right intrahepatic bile duct on CT scan (Fig. 3A). On MRI, this lesion depicted as high SI on T2WI, and low SI on T1WI. After, contrast administration, it showed arterial enhancement. It showed washout on portal and delayed phase. And low SI on hepatobiliary phase, and definite diffusion restriction (Fig. 3B-H). This lesion caused biliary obstruction, resulted in peripheral intrahepatic bile duct dilatation. And the serum total 


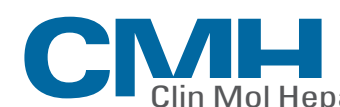

Volume 22 Number_1 March 2016
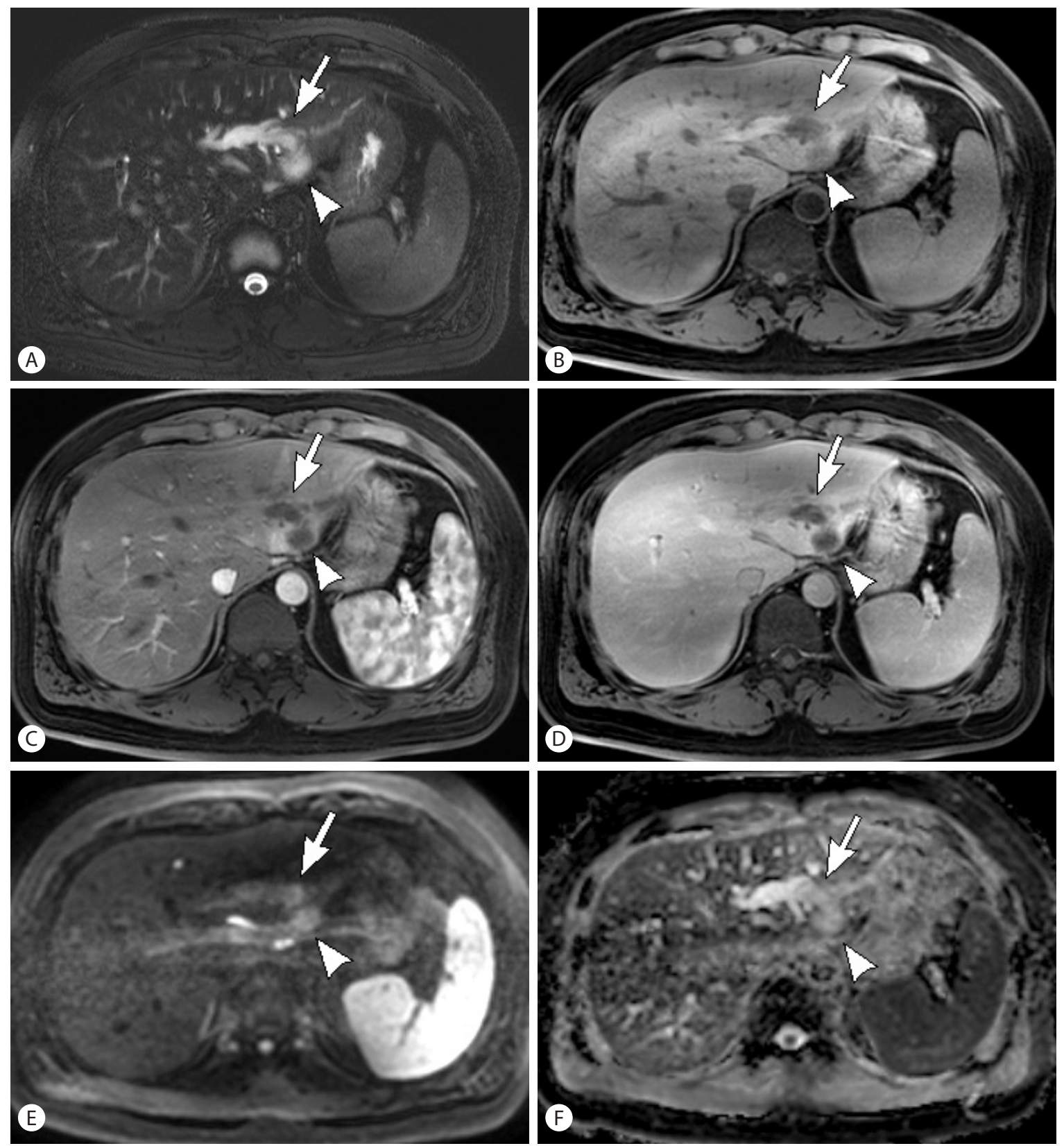

Figure 2. On MRI, intraductal lesion (white arrows) and periductal mass (white arrowheads) show high SI on T2WI (A), and low SI on T1WI (B). There is no avid enhancement on arterial phase images (C) and persistent low SI on delayed phase images (D). The lesion demonstrates diffusion restriction (E, F). After left lateral segmentectomy, this lesion was proven to be metastatic adenocarcinoma from colon. MRI, magnetic resonance imaging; SI, signal intensity; T2WI, T2-weighted images; T1Wl, T1-weighted images.

bilirubin level was $17.2 \mathrm{~mL} / \mathrm{dL}$, and direct bilirubin was $12.9 \mathrm{~mL} /$ dL. Our impression was intraluminal growing hilar cholangiocarcinoma. The patient underwent right hemihepatectomy and caudate lobectomy. However, the histopathologic diagnosis was intraductal growing hepatocellular carcinoma (HCC), confined to the bile duct wall.

\section{DISCUSSION}

\section{Intrabiliary metastases}

Morphologically, the gross appearance of liver metastases from colorectal carcinoma usually form as nodular or round masses, whereas intrabiliary growth in the bile duct is a rare manifesta- 

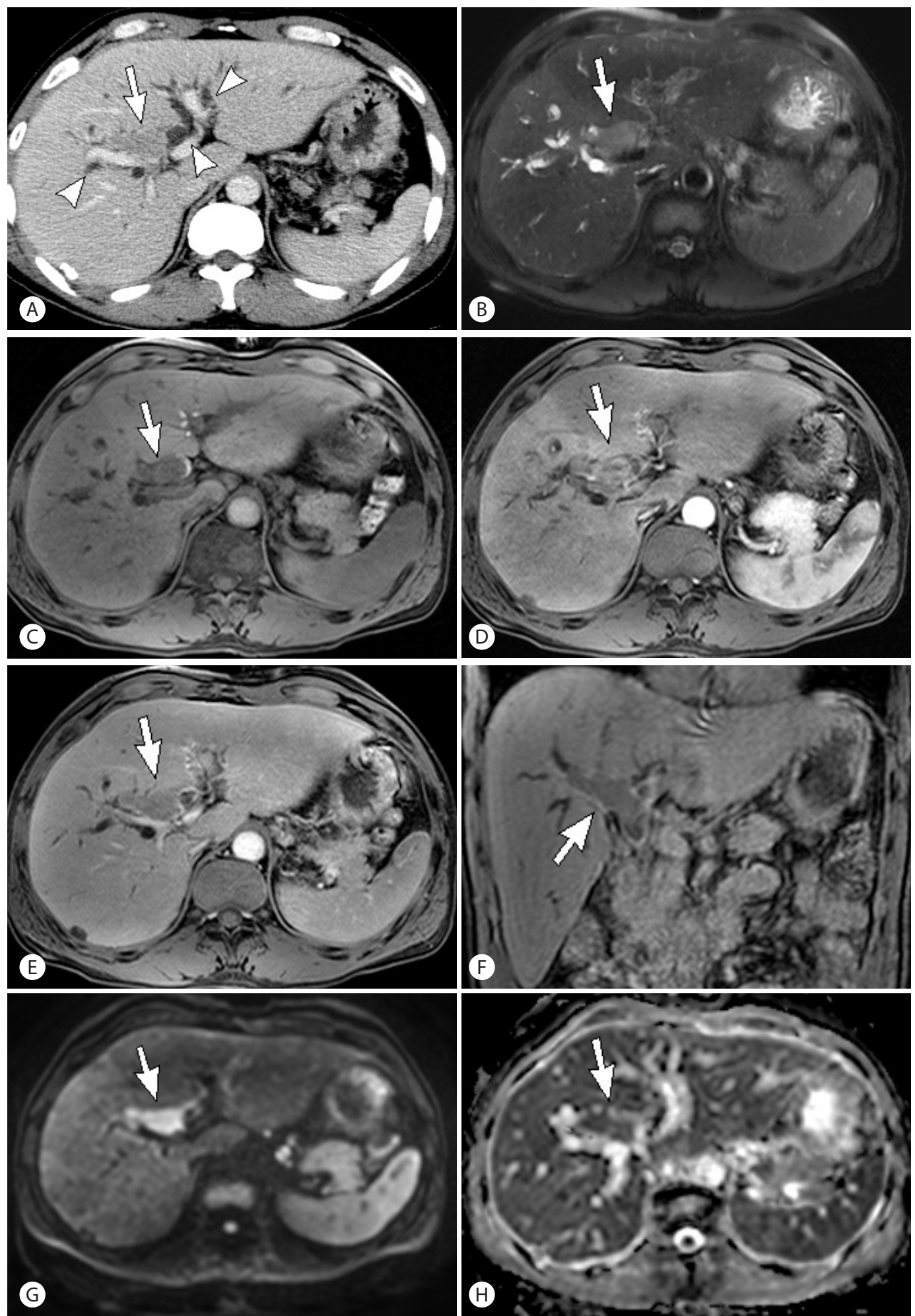

Figure 3. Intraductal growing polypoid enhancing mass (white arrows) at the proximal bile duct with peripheral intrahepatic bile duct dilatation (white arrowheads) on CT scan (A). On MRI, lesion shows high SI on T2WI (B), and low SI on T1WI (C). It shows arterial enhancement (D), low SI on delayed and hepatobiliary phase (E, F), and diffusion restriction $(\mathrm{G}, \mathrm{H})$. Right hemihepatectomy and caudate lobectomy was done, and the histopathologic diagnosis was intraductal growing HCC. CT, computed tomography; MRI, magnetic resonance imaging; SI, signal intensity; T2WI, T2-weighted images; T1WI, T1-weighted images; HCC, hepatocellular carcinoma. 
tion. ${ }^{1,2}$ Primary tumors that metastasize to the bile ducts include cancers of the lung, breast, gallbladder, colon, testicle, prostate, or pancreas; melanoma; and lymphoma. Of the listed cancers, colon adenocarcinoma, on account of its proclivity to spread along epithelial surfaces, shows a higher predilection to involve the biliary ducts. ${ }^{1}$ The mechanism of intrabiliary metastasis of colon cancer is unclear; however, possible mechanisms include vascular invasion via the lymph vessels, blood vessels, or biliary tract, and implantation of malignant cells to the bile duct epithelium via the bile stream. $^{3}$

Imaging findings of metastases that show intrabiliary growth may mimic cholangiocarcinoma or IPNB. ${ }^{1,4-6}$ When an intraductal lesion was detected, the following findings were found to be suggestive of intraductal metastasis, rather than intraductal cholangiocarcinoma: colorectal cancer as the extra-biliary malignant disease, a parenchymal mass that was contiguous with the intraductal lesion, and expansile growth of the intraductal lesion. ${ }^{7}$ According to the previous report, usually, intraductal metastasis exhibits expansile intraluminal extension, but intraductal cholangiocarcinoma exhibits intraepithelial extension with a papillary growth pattern, due to its origination from the underlying papilloma or adenoma. ${ }^{7}$ However, there are no reliable imaging features for distinction of biliary metastases from cholangiocarcinomas or IPNBs; ${ }^{8}$ therefore, diagnosis is based solely on history and histologic findings.

Immunohistochemical staining using cytokeratin (CK)-7 and CK20 and mucin is necessary to differentiate between metastatic lesion arising from a colon carcinoma and cholangiocarcinoma or IPNB; metastatic colon carcinoma shows CK20 positivity and CK7 negativity in contradistinction to the adjacent bile duct epithelium, which is CK7 positive and CK20 negative. ${ }^{9}$

In short, we should take metastatic lesions into consideration, when an intraductal lesion is found in a patient with colorectal malignancy, or a history of colorectal surgery.

\section{Intraductal growing hepatocellular carcinoma}

Hepatocellular carcinoma is the most common primary malignant hepatic neoplasm and rarely may show biliary ductal growth or invasion, as previously reported incidence of 1.2-9\%. ${ }^{10,11}$ These types have been classified as icteric type HCC by Lin et al. ${ }^{12}$ These tumors may manifest obstructive jaundice as the initial complaint. ${ }^{13}$ Identification of this group of patients is clinically important, because surgical treatment may be beneficial. However, accurate preoperative diagnosis is difficult owing to the low prevalence of this pattern of growth and the nonspecific imaging findings.

The imaging finding of HCC with an intraductal growth pattern appears as expansile soft tissue masses that cause dilatation of the bile duct. ${ }^{13}$ According to the previous report, significant variables that help to differentiate HCC with intraductal growth from intraductal cholangiocarcinoma include the presence of parenchymal mass, liver cirrhosis, and a hyperattenuating intraductal tumor on the hepatic arterial phase. ${ }^{14}$ The presented case in this report, intraductal tumor shows hyperenhancement on the arterial phase and washout on the portal venous phase compared to the liver parenchyma. Clinical and laboratory data such as the presence of predisposing factors for cirrhosis such as positive serologic results for hepatitis $B$ or $C$ and high serum levels of AFP may help to suggest the diagnosis of HCC.

\section{SUMMARY}

Despite remarkable recent improvements in the imaging tools for diagnosis of liver lesions, rare bile duct tumors are sometimes incorrectly diagnosed as cholangiocarcinoma or IPNB. To diagnose correctly and lead to proper treatment option, doctors should be familiar with the clinical manifestation and the imaging findings of rare bile duct tumors such as intraductal metastasis or intraductal growing HCC as well as more common tumors such as cholangiocarcinoma.

\section{Conflicts of Interest}

The authors have no conflicts to disclose.

\section{REFERENCES}

1. Riopel MA, Klimstra DS, Godellas CV, Blumgart LH, Westra WH. Intrabiliary growth of metastatic colonic adenocarcinoma: a pattern of intrahepatic spread easily confused with primary neoplasia of the biliary tract. Am J Surg Pathol 1997;21:1030-1036.

2. Yasui K, Hirai T, Kato T, Torii A, Uesaka K, Morimoto T, et al. A new macroscopic classification predicts prognosis for patient with liver metastases from colorectal cancer. Ann Surg 1997;226:582-586.

3. Yamao T, Hayashi $H$, Higashi T, Takeyama H, Kaida T, Nitta $H$, et al. Colon cancer metastasis mimicking intraductal papillary neoplasm of the extra-hepatic bile duct. Int J Surg Case Rep 2015;10:91-93.

4. Nanashima A, Tobinaga S, Araki M, Kunizaki M, Abe K, Hayashi H, et al. Intraductal papillary growth of liver metastasis originating 
Ah Yeong Kim, et al. Intraductal malignant tumors

from colon carcinoma in the bile duct: report of a case. Surg Today 2011:41:276-280.

5. Okano K, Yamamoto J, Moriya Y, Akasu T, Kosuge T, Sakamoto $M$, et al. Macroscopic intrabiliary growth of liver metastases from colorectal cancer. Surgery 1999;126:829-834.

6. Takamatsu S, Teramoto K, Kawamura T, Kudo A, Noguchi N, Irie T, et al. Liver metastasis from rectal cancer with prominent intrabile duct growth. Pathol Int 2004;54:440-445.

7. Lee DH, Ahn YJ, Shin R, Lee HW. Metastatic mucinous adenocarcinoma of the distal common bile duct, from transverse colon cancer presenting as obstructive jaundice. Korean J Hepatobiliary Pancreat Surg 2015;19:125-128.

8. Menias CO, Surabhi VR, Prasad SR, Wang HL, Narra VR, Chintapalli $\mathrm{KN}$. Mimics of cholangiocarcinoma: spectrum of disease. Radiographics 2008;28:1115-1129.

9. Tokai H, Kawashita Y, Eguchi S, Kamohara Y, Takatsuki M, Okudaira $\mathrm{S}$, et al. A case of mucin producing liver metastases with intrabiliary extension. World J Gastroenterol 2006;12:4918-4921.

10. Kojiro M, Kawabata K, Kawano Y, Shirai F, Takemoto N, Nakashima T. Hepatocellular carcinoma presenting as intrabile duct tumor growth: a clinicopathologic study of 24 cases. Cancer 1982;49:2144-2147.

11. Leong JW, Ho JM, Ng HS, Raj JP. Early hepatocellular carcinoma presenting with biliary ductal invasion-a case report. Ann Acad Med Singapore 2000;29:101-104.

12. Lin TY, Chen KM, Chen YR, Lin WS, Wang TH, Sung JL. Icteric type hepatoma. Med Chir Dig 1975;4:267-270.

13. Qin LX, Tang ZY. Hepatocellular carcinoma with obstructive jaundice: diagnosis, treatment and prognosis. World J Gastroenterol 2003;9:385-391.

14. Jung AY, Lee JM, Choi SH, Kim SH, Lee JY, Kim SW, et al. Computed tomography features of an intraductal polypoid mass: differentiation between hepatocellular carcinoma with bile duct tumor invasion and intraductal papillary cholangiocarcinoma. J Comput Assist Tomogr 2006;30:18-24. 